\title{
Eating disorder/and or eating behavior researches from West Bengal, India: Suppressing the facts??
}

\author{
Arup Ratan Bandyopadhyay \\ Professor, Department of Anthropology, University College of Science, Technology \& Agriculture, University of Calcutta, \\ Kolkata, West Bengal, India
}

\section{A B S T R A C T}

\begin{abstract}
The present attempt is to review the research articles concerning to eating disorder/and or eating behavior on anthropological approach from West Bengal, India. This review, focused on the five published research work (between 2014 and 2016) funded by Biological Anthropology unit of the Statistical Institute, Kolkata India and a review article (funded by University of Calcutta). Most of the studies, concerned with socio demographic concomitants particularly, and suppressed the facts of the major issues related to the well known insight of genetics of eating disorder/and or eating behavior. Moreover, the review article (2016) on Eating Behaviours and Body Weight Concerns among Adolescent Girls attempted to find out the probable factors of Eating Behaviours and Body Weight Concerns but surprisingly suppressed and or overlooked the efforts of the researches concerning the genetic polymorphism on eating behaviours and body weight concerns among the adolescent. Most of the research article failed to justify the research article title and finally ended up with much known facts, Furthermore, all the research articles also suppressed the facts of underlying genetic factors published much earlier. Under this circumstances, the present review is the dual endeavor firstly, earnest plead to the researcher to avoid the suppression of facts concerning research on eating disorder/and or eating behavior and on the other hand, to sketch a probable direction of the research, for the scholars, who might have inclination to undertake such an important research issue in this century.
\end{abstract}

Access this article online

Website:

http://nepjol.info/index.php/AJMS

DOI: 10.3126/ajms.v8i2.16395

E-ISSN: 2091-0576

P-ISSN: 2467-9100

Key words: Eating behavior, Eating disorders, Heritability, Over simplification,

Suppression of facts

\section{INTRODUCTION}

Eating behavior is a complex trait with both genetic and environmental influences. The eating disorders anorexia nervosa (AN) and bulimia nervosa (BN) traditionally have been viewed as socio-cultural in origin. However, recent behavioral genetic findings suggest substantial genetic influence on these disorders. ${ }^{1}$ With the advent of human genome project and its completion certainly vindicated, that the etiology of these disorders is presumed to be influenced by developmental, social, and biological processes. ${ }^{2}$ Cultural attitudes toward thinness have relevance to the psychopathology of eating disorders, but they are unlikely to be sufficient to account for the pathogenesis of these disorders. Notably, dieting behavior is quite common in industrialized countries throughout the world, yet $\mathrm{AN}$ and $\mathrm{BN}$ affect only an estimated 0.3 to 0.7 percent, and 1.7 to 2.5 percent, respectively, of females in the general population. ${ }^{3}$ Moreover, numerous descriptions of $\mathrm{AN}$ dated from the middle of the 19th century suggesting that factors other than modern culture play an etiologic role. In addition, both syndromes have a relatively homogeneous clinical presentation, sex distribution, and age-of-onset, supporting the possibility of some biological susceptibility. This is not to discount the role of culture, as the introduction of Western ideals of thinness may serve to release a biological propensity toward eating disorders ${ }^{4}$ possibly by increasing behaviors, 
such as dieting, that may trigger the spiral of disordered eating. Recent findings from behavior genetic studies suggest that this biological vulnerability might be genetic in nature. The rapid rise in obesity and associated co-morbidities (metabolic syndrome, coronary artery disease, sleep apnea, skeletal disorders, hyperlipidemia, and hypertension) over the past 30 years has led to the urgency of coming to a more complete understanding of the pathophysiology of obesity. ${ }^{5}$ The study of eating behavior attempts to define eating patterns and food preferences, and to explain why there is gravitation toward specific behaviors and food choices? And also aims to develop approaches to bring about effective changes in modifiable behaviors. Knowledge of the biological mechanisms guiding eating behavior can provide effective treatment targets for obesity and associated disorders. ${ }^{6}$ A widely used research tool known as the Three-Factor Questionnaire (TFQ) has been used to quantify eating behaviors in normal-weight, obese, and in individuals with eating disorders. ${ }^{7}$ This questionnaire uses a series of questions to measure three patterns of behavior: restraint, disinhibition, and hunger and subsequently on the basis of heritability and linkage analysis of eating behavior measured by the TFQ provides evidence that these behavior traits are heritable. ${ }^{8,9}$ Furthermore, GAD (glutamic acid decarboxylase) has also been linked to eating behavior. GAD decarboxylates glutamate into GABA ( $\gamma$-aminobutyric acid), a major inhibitory neurotransmitter in the brain. Two specific GAD variants, rs 7908975 and rs 992990 have been reported to be associated with disinhibition and disordered food intake, specifically increased carbohydrate intake, in women. ${ }^{10}$ Few more aspects are also related in this context, such as genetics of taste affects food preference and food intake thereby directly influencing eating behavior. ${ }^{11}$ Bitter, sweet and umami tastes are mediated by G-protein-coupled receptors (GPCR) are encoded by 25-30 TAS2R genes, located on chromosomes 12p13, 7q34 and 5p15.31. The ligand specificity of TAS2Rs appears to be quite broad, consistent with their roles in detecting thousands of bitter-tasting compounds. ${ }^{12}$ Genetic variation in TAS1R and TAS2Rs may impact eating behavior via altered taste perception as well as via alteration in neuroendocrine signals impacting satiety. The observation that these receptors are involved in both taste and secretion of hormones involved in satiety tell that these processes are biologically entwined. ${ }^{6}$

Research into meal size and selection is especially complex as socioeconomic environment, learned eating behaviors, physiologic conditions, meal quantity, frequency, and timing are thought to be at least in part under genetic control. The study of genetic variants in digestive neuroendocrine hormones, such as CCK, leptin and ghrelin, are providing new insights into how these hormones and their genetic variants may be involved in pathways regulating appetite and eating behavior. The current knowledge with respect to genetic variants linked to meal selection and size contributing to eating behaviors like, extreme snacking behavior, ${ }^{13}$ binge eating. ${ }^{14} \mathrm{~A}$ variety of cognitive pathways are involved in motivation and control of eating behavior. Eating behavior research utilizing FMRI has focused on blood oxygen level dependence (BOLD) changes in specific brain regions and demonstrated FMRI has been used successfully to shed light into pathways involved in eating behavior. ${ }^{15}$ The concurrent use of neuroimaging techniques, specifically functional magnetic resonance imaging (FMRI) demonstrated specific neural reactions in response to food stimulus is revolutionizing the study of eating behavior. Furthermore, varieties of cognitive pathways were found to be involved in motivation and control of eating behavior. Combination of genetic studies with FMRI to investigate eating behavior modalities helping to uncover interrelationships among genetics and the neurophysiologic pathways involved in food response and eating behavior. It is hypothesized that polymorphisms in genes involved in the neurophysiology of feeding and reward processing would demonstrate differential responses in brain regions known to be involved in food reward. ${ }^{16}$

FTO, fat mass and obesity-associated gene, has been highly associated with increased risk of obesity. FTO, (chromosome 16) and is expressed in adipocytes, the pancreas, and the hypothalamus, particularly in regions known to regulate appetite. ${ }^{17}$ FTO variant confers a predisposition to obesity and may play a role in the control of food intake (eating behavior) and food choice, perhaps involving a hyperphagic phenotype or a preference for energy-dense foods. ${ }^{18}$

On the basis of above mentioned context, it is most likely, that without considering gene involvements for eating disorder/and or behavior research but only considering socio demographic concomitants would generate misleading and futile research which ultimately ended up with much known facts and as well as consumption of the grants without effectiveness of the deliverables to the society. In this context, the publication ${ }^{19}$ regarding eating behaviors under the terminology of "eating and weight concerns", "eating behaviors and body weight", "body weight concerns among urban adolescent Girls: A Microlevel Study" and "Weight concerns and food habits of adolescent girls in two contrasting ecological regions" seems to be misleading, since issues regarding the earlier and contemporary researches on gene involvement have been completely suppressed and unacknowledged for unknown reasons. The above 
mentioned research areas (eating behavior/and or eating disorder) were mostly funded by Biological Anthropology Unit of Indian Statistical Institute, Kolkata and the entire research article only considered the socio demographic aspects. The examples can be cited form the research paper on "Eating and weight concerns among Sikkimese adolescent girls and their biocultural correlates: an exploratory study ${ }^{\prime 19}$ under the funding of Indian Statistical Institute could raise the questions on one of the objectives as "eating behavior modifications" which ultimately ended with the result as "Girls from families with a bigher economic status are about two times more likely to report dissatisfaction with their body weight". Despite of the title of the publication ${ }^{19}$ starts on "eating" and finally confused with food choice on the basis of "principal component analysis is categorized as 'vegetable-fruit-milk-cereals', 'meat-fish-egg' and 'snacks-ice cream-beverages'. It perhaps becomes dangerous trend to use "statistical test juggleries", which ultimately obtain the results which not at all compatible with the objectives of the study ${ }^{19}$ and finally ended up with a generalized much known outcome as "A directional trend has not been found in the present study between economic status and food groups." In fact, no reason and explanation have been found from the study to evaluate from the narration as "However, more married girls, irrespective of their age, showed significantly lower scores for the same food group (snacks-ice cream-beverages) than their unmarried counterparts" from again it proved the unnecessary "statistical test juggleries". Moreover, the values for anthropometric measures is not at all clear whether they are incidences and if incidence, why t tests has been done? Finally although the title of the paper depicted "biocultural correlates" but in the entire text surprisingly no issues on biocultural correlates were addressed!!!

It is noteworthy, that eating disorder symptoms themselves also appear to be moderately heritable. Twin studies of binge eating, self-induced vomiting, and dietary restraint suggest that these behaviors are roughly 46 to 72 percent heritable. ${ }^{20,21}$ Likewise, pathological attitudes such as body dissatisfaction, eating and weight concerns, and weight preoccupation, revealed heritability of roughly 32 to 72 percent. ${ }^{22}$ Although it is fairly established in 1990 s about the heritability of body dissatisfaction, eating and weight concerns, but the publication ${ }^{19}$ on "exploratory study" after a decade, surprisingly authors seems to be unaware or deliberately set aside the important aspects of heritability vis $-a$ vis the genetics which is beyond to the publication ethics and as well as suppressing the facts.

Another endeavor, utilizing the grant of University of Calcutta pertaining to the review article ${ }^{23}$ entitled "Eating Behaviours and Body Weight Concerns among Adolescent Girls" contains 115 references. Although, authors claimed in abstract "probable factors underlying it" but, unexpectedly the entire endeavor surprisingly suppressed the facts of common gene polymorphisms for eating behaviors in children and adolescents ${ }^{17}$ and as well as the heritability concern of "Eating Behaviours and Body Weight Concerns" as underlying probable factors. This might be due to keep away themselves (authors) from the facts of genetics and at the same time misleading and oversimplifying the issues of Eating Behaviours and Body Weight Concerns. The review finally found "Factors Infuencing Eating Behaviors and Body Weight Concerns" as "Family Environment and Peer Pressure, Sociocultural and Economic Context, Concern over Body Image, Gender and Age, and Eating Away from Home", with no appreciation to the earlier research work on probable factors of genetics on Eating Behaviors. This unfortunate phenomenon probably happened only to increase numbers of publication for unknown reasons and might be for API scores by paying huge Article Processing Charge (APC) (\$600 -http://www.hindawi.com/journals/aph/apc/) from the Calcutta University research grant to a misleading and inadequate review and as well as misleading claimant as research personnel in this area only for public display and most seriously beyond the publication ethics.

Yet again, Indian Statistical Institute funded research work ${ }^{24}$ from Biological Anthropology unit (APC \$500, http:// www.hindawi.com/journals/janthro/apc/) on "Body Weight Concerns among Urban Adolescent Girls: A Microlevel Study". Unfortunately, once again did not address the genetics of body weight concerns as mentioned above. Surprisingly, the paper, revealed unusual (not related to the title) association of "sociodemographic variables as predictors of different issues related to dietary practices." and found close association $\mathrm{R}^{2}$ ranging from $0.02-0.09$, while, heritability estimated in twin studies roughly as 32 to 72 percent for body dissatisfaction, eating and weight concerns ${ }^{1}$ and twin studies often focus on broader phenotypes or subthreshold eating disorders. These studies consistently yielded moderate to substantial heritability. ${ }^{25}$ Furthermore, the publication attracts question regarding "Microlevel study", which remain unanswered, Why this is micro level study? Finally the paper did not concern entirely the genetics of "Body Weight Concerns", might be due to inability to address or purposely avoided the aspects of genetics which is again misleading and suppressions of facts in this urgent research area and beyond the publication ethics.

Another example of recent publication ${ }^{26}$ funded by Indian Statistical Institute (Biological Anthropology Unit), paper entitled "Weight concerns and food habits of adolescent girls in two contrasting ecological regions: A comparative study in India". Understanding the "weight concern" it has been adapted and slightly modified from the "four questioned" questionnaires used 
in other western studies with dichotomous response choices (yes or no) measured the cognitive and emotional aspects of weight related issues among girls". Surprisingly, the first question as mentioned in the article was "How do they perceive their own body weight?" which seems to be entirely difficult to dichotomous response choices (yes or no). Finally, this paper ended with the very much known facts, "Diversity in culture, ethnicity and socio-economic standard perbaps develop the disparity in food habits and weight concerns between girls of urban billy areas of Sikkim and the city of Kolkata" as conclusion. Concerning APC, it was found to be USD 1800 (http://cdn.elsevier. $\mathrm{com} /$ promis_misc/j.custom 97.pdf). Moreover, two of the authors ${ }^{26}$ are attached to Biological Anthropology Unit and unfortunately failed to provide any contemporary biological anthropology acumen in this research article. It is evident that these researches on eating disorders/and or eating behavior seems to be misleading and eventually suppressing the facts by consuming the research grants of esteemed Institution like Indian Statistical Institute. Moreover, authors seem to be yet again unaware and of the established facts with evidence published in 2005 for high expression of FTO gene in the brain ${ }^{27}$ and its relation with eating behavior and the association of genetic variation.

Yet again, the publication ${ }^{28}$ with financial support from a grant of the Indian Statistical Institute to the Biological Anthropology Unit, entitled "Body weight and body shape concerns and related behaviours among Indian urban adolescent girls" and again overlooked and failed to address the aspects of heritability of body weight and body shape concern, since, increased heritability in postpubertal relative to prepubertal twins who were the same age provided strong evidence of potential pubertal activation of the heritability of eating pathology that may be mediated by ovarian hormones. These findings are important for highlighting not only developmental differences in genetic effects, but also the potential role of ovarian steroids in the heritability of these disorders. ${ }^{1}$ Moreover, Cultural attitudes toward thinness have relevance to the psychopathology of eating disorders, but they are unlikely to be sufficient to account for the pathogenesis of these disorders. However, the earlier publications ${ }^{29}$ demonstrated body weight is highly heritable leading to questions of shared genetic transmission between body weight and eating pathology. Earlier study directly examined ${ }^{20}$ this question by investigating shared genetic transmission of BMI and disordered eating, including body dissatisfaction, weight preoccupation, overall eating pathology, and the use of compensatory behaviors, such as self-induced vomiting and laxative abuse and findings suggested some shared genetic transmission, although again, the majority of genetic influence on these disordered eating variables was independent of the genes influencing BMI. The above mentioned research article ${ }^{26}$ from Indian Statistical Institute, Kolkata found the result as "The likelihood of adopting unhealthy eating practices was significantly higher among overvveight than normal-weight girls." But studies ${ }^{30}$ related to genetic variants in digestive neuroendocrine hormones, such as CCK, leptin and ghrelin (located on chromosome 3 and 28-amino acid peptide), had already been provided new insights into how these hormones and their genetic variants may be involved in pathways regulating appetite and eating behavior including involvement in promoting meal intake and hunger through receptors ${ }^{31}$ in the hypothalamus. Plasma ghrelin levels rise pre-meal and are suppressed by food intake. GHRL is the gene product is involved in growth hormone release, and post translational modifications yield the hormones ghrelin and obstatin. Obstatin opposes the effects of ghrelin and is responsible for satiety and decreasing food intake. ${ }^{32}$ Many studies have been devoted to investigating GHRL variants with respect to obesity. A common variant, Leu72Met has been associated with obesity, ${ }^{33,34}$ metabolic syndrome, ${ }^{35}$ and binge eating. ${ }^{14}$ Leptin and CCK work in opposition to ghrelin to promote satiety. CCK is released ${ }^{35}$ in response to lipids and promotes rapid post-prandial satiety in contrast to the long term action of leptin. In a large case-control study $^{13}$ of obese and normal weight women, common leptin variants, (rs4577902, rs2060736, and rs4731413), have been found to be associated with increased risk of extreme snacking behavior (top 5th percentile based on 11 question questionnaire), but not increased meal size. CCK variants (rs6809785, rs7611677, rs6801844, and rs6791019) were found to be more associated with extreme meal size, but not increased snacking behavior in the same study. This study ${ }^{13}$ suggests that genetic variation in genes encoding CCK and leptin may contribute to obesity risk by influencing satiety. However, the research $\operatorname{article}^{27}$ surprisingly overlooked the earlier research efforts regarding genetic variation to body weight and body shape. At the same time consecutive publications $s^{19,23,24,26,28}$ on this urgent area unfortunately made too much over simplified and misleading related to unhealthy diet and not provided any rightful appreciation of the above mentioned genetic contributions and contravened the publication ethics.

In this circumstance, it become very misleading and treacherous condition in this urgent area of research due to globalization on eating disorder and different terms like, weight concern, in the West Bengal, India scenario by the research undertaken mostly funded by Indian Statistical Institute (Biological Anthropology Unit) including University of Calcutta. This over simplification and misleading research work might hamper the future of young researcher involved in this important area of research for their days ahead in professional anthropology. 
It could be anticipated the research work on Eating disorder/ and or eating behavior will give proper justice in future publications towards mentioning the involvements of the genetic polymorphisms and as well as make an end of this over simplification and misleading research works which ultimately ended up to much known facts after consumption of grants from esteemed institution like, University of Calcutta and Indian Statistical Institute.

\section{ACKNOWLEDGEMENT}

The author gratefully acknowledge the financial support of University of Calcutta [BI 65 (9)].

\section{REFERENCES}

1. Berrettini W. The Genetics Eating Disorders. Psychiatry 2004; Nov: $19-25$.

2. Garner DM. Pathogenesis of anorexia nervosa. Lancet 1993, 341:1631-1635.

3. American Psychiatric Association. Diagnostic and Statistical Manual of Mental Disorders DSM-IV. Washington, DC 1994.

4. Becker A. Eating disorders in Fiji. Proc. American Psychiatric Association meeting. Washington, DC 1999.

5. Murray S, Tulloch A, Gold MS and Avena NM. Hormonal and neural mechanisms of food reward, eating behaviour and obesity. Nature Reviews Endocrinology 2014; 10: 540-552.

6. Grimm ER and Steinle NI. Genetics of Eating Behavior, Established and Emerging Concepts. Nutr Rev 2011, 69: 52-60.

7. Stunkard AJ, Harris JR, Pedersen NL and McClearn GE. The body-mass index of twins who have been reared apart. N Engl J Med 1990; 32: 1483-1487.

8. Bouchard L, Drapeau V, Provencher V, Lemieux S, Chagnon Y, Rice T, et al. Neuromedin beta, a strong candidate gene linking eating behaviors and susceptibility to obesity. Am J Clin Nutr 2004; 80:1478-1486.

9. Steinle N, Pollin T, O'Connell J, Mitchell B and Shuldiner A. Variants in the ghrelin gene are associated with metabolic syndrome in the Old Order Amish. J Clin Endocrinol Metab 2005; 90: 6672-6677.

10. Choquette AC, Lemieux S, Tremblay A, Drapeau V, Bouchard C, Vohl MC, et al. GAD2 gene sequence variations are associated with eating behaviors and weight gain in women from the Quebec family study. Physiol Behav 1998; 4:505-510.

11. Rolls ET. Taste, olfactory, and food texture processing in the brain, and the control of food intake. Physiol Behav 2005; 85:45-56.

12. Scott K. Taste recognition, food for thought. Neuron 2005, 48:455-464

13. de Krom M, van der Schouw YT, Hendriks J, Ophoff RA, van Gils $\mathrm{CH}$, Stolk RP, et al.Common genetic variations in CCK, leptin, and leptin receptor genes are associated with specific human eating patterns. Diabetes 2007; 56: 276-280.

14. Monteleone P, Tortorella A, Castaldo E, Di Filippo C and Maj M. The Leu72Met polymorphism of the ghrelin gene is significantly associated with binge eating disorder. Psychiatric Genetics 2007; 17:13-16.

15. Haase L, Cerf-Ducastel B and Murphy C. Cortical activation in response to pure taste stimuli during the physiological states of hunger and satiety. Neuroimage 2009; 44:1008-1021.
16. Felsted JA, Ren X, Chouinard-Decorte $F$ and Small DM. Genetically determined differences in brain response to a primary food reward. J Neurosci 2010; 30: 2428-2432.

17. Cecil J, Dalton M, Finlayson G, Blundell J, Hetherington M and Palmer C. Obesity and eating behaviour in children and adolescents, contribution of common gene polymorphisms. Int Rev Psychiatry 2010; 24:200-210.

18. Tanofsky-Kraff M, Han JC, Anandalingam K, Shomaker LB, Columbo KM, Wolkoff LE, et al. The FTO gene rs9939609 obesity-risk allele and loss of control over eating. Am J Clin Nutr 2009; 90: 1483-1488

19. Mishra SK and Mukhopadhyay S. Eating and weight concerns among Sikkimese adolescent girls and their biocultural correlates, an exploratory study. Pub Health Nutri 2010; 14: 853-859.

20. Klump KL, Bulik CM, Pollice C, Halmi KA, Fichter MM, Berrettini WH, et al.Temperament and character in women with anorexia nervosa. J Nerv Ment Dis 2000; 188: 559-567.

21. Bulik CM, Sullivan PF and Kendler KS. The Genetic epidemiology of binging and vomiting. $\mathrm{Br}$ Journal of Psychiatry 1998; 173:75-79.

22. Wade T, Martin NG, Neale MC, Tiggemann M, Treloar SA, Bucholz KK, et al.The structure of genetic and environmental risk factors for three measures of disordered eating. Psychol Med 1999; 29:925-934.

23. Mallick N, Ray S and Mukhopadhyay S. Eating Behaviours and Body Weight Concerns among Adolescent Girls. Advances in Public Health 2014; Article ID 257396, 8 pages.

24. Mukhopadhyay S, Ganguly N and Mishra SK. Body Weight Concerns among Urban Adolescent Girls, A Microlevel Study. Journal of Anthropology 2014; Article ID 264925, 6 pages

25. Slof-Op 't Landt MC, van Furth EF, Meulenbelt I, Slagboom PE, Bartels M, Boomsma DI, et al. Eating disorders, from twin studies to candidate genes and beyond. Twin Res Hum Genet 2005; 8:467-482.

26. Som N, Mishra SK and Mukhopadhyay S. Weight concerns and food habits of adolescent girls in two contrasting ecological regions, A comparative study in India. Eating Behaviors 2016; 20: 21-26.

27. Velders FP, De Wit JE, Jansen PW, Jaddoe VW, Hofman A, Verhulst FC, et al. FTO at rs9939609, food responsiveness, emotional control and symptoms of ADHD in preschool children. PLoS One 2012; 7:,e49131.

28. Som $\mathrm{N}$ and Mukhopadhyay $\mathrm{S}$. Body weight and body shape concerns and related behaviours among Indian urban adolescent girls. Public Health Nutrition 2014; 18: 1075-1083.

29. Stunkard AJ and Messick S. The three-factor eating questionnaire to measure dietary restraint, disinhibition and hunger. J Psychosom Res 1985; 29:71-83.

30. Nakazato M, Murakami N, Date $Y$, Kojima M, Matsuo $H$, Kangawa $\mathrm{K}$, et al. A role for ghrelin in the central regulation of feeding. Nature 2001; 409:194-198.

31. Callahan HS, Cummings DE, Pepe MS, Breen PA, Matthys CC and Weigle DS. Postprandial suppression of plasma ghrelin level is proportional to ingested caloric load but does not predict intermeal interval in humans. J Clin Endocrinol Metab 2004; 89: 1319-1324.

32. Zhang JV, Ren PG, Avsian-Kretchmer O, Luo CW, Rauch R, Klein $\mathrm{C}$, et al. Obestatin, a peptide encoded by the ghrelin gene, opposes ghrelin's effects on food intake. Science 2005; 310:996-999.

33. Korbonits M, Gueorguiev M, O'Grady E, Lecoeur C, Swan DC, Mein CA, et al. Variation in the ghrelin gene increases weight and decreases insulin secretion in tall, obese children. J Clin Endocr Metab 2002; 87:4005-4008. 
34. Hinney A, Hoch A, Geller F, Schäfer H, Siegfried W, Goldschmidt H, et al. Ghrelin gene, identification of missense variants and a frameshift mutation in extremely obese children and adolescents and healthy normal weight students. J Clin Endocr Metab 2002; 87: 2716-2719.

35. Steinle NI, Hsueh WC, Snitker S, Pollin TI, Sakul H, St Jean PL, et al. Eating behavior in the Old Order Amish, heritability analysis and a genome-wide linkage analysis. Am J Clin Nutr 2002; 75:1098-1106.

\section{Website:}

http://cdn.elsevier.com/promis_misc/j.custom97.pdf (accessed on 14/01/2017)

http://www.hindawi.com/journals/aph/apc/ accessed on 14/01/2017)

http://www.hindawi.com/journals/janthro/apc/(accessed on $14 / 01 / 2017)$.

Authors Contribution:

It is a single author contribution and review article.

Source of Support: University of Calcutta, BI 65 (9), Conflict of Interest: None declared. 